\title{
Task-Free Functional Language Networks: Reproducibility and Clinical Application
}

\author{
Giovanni Battistella, ${ }^{1}$ Valentina Borghesani, ${ }^{1}$ Maya Henry, ${ }^{1,2}$ Wendy Shwe, ${ }^{1}$ Michael Lauricella, ${ }^{1}{ }^{\circledR}$ Zachary Miller, ${ }^{1}$ \\ Jessica Deleon, ${ }^{1}$ Bruce L. Miller, ${ }^{1}$ Nina Dronkers, ${ }^{3}$ Simona M. Brambati, ${ }^{4}{ }^{\circ}$ William W. Seeley, ${ }^{1}$ Maria Luisa Mandelli, ${ }^{1}$ \\ and Maria Luisa Gorno-Tempini ${ }^{1}$ \\ ${ }^{1}$ Memory and Aging Center, Department of Neurology, University of California, San Francisco, California 94158, ${ }^{2}$ Department of Communication Sciences \\ and Disorders, University of Texas, Austin, Texas 78712, ${ }^{3}$ Department of Psychology, University of California, Berkeley, California 94720, and \\ ${ }^{4}$ Département de Psychologie, Université de Montréal, Quebec H3T 1J4, Canada
}

Intrinsic connectivity networks (ICNs) identified through task-free fMRI (tf-fMRI) offer the opportunity to investigate human brain circuits involved in language processes without requiring participants to perform challenging cognitive tasks. In this study, we assessed the ability of tf-fMRI to isolate reproducible networks critical for specific language functions and often damaged in primary progressive aphasia (PPA). First, we performed whole-brain seed-based correlation analyses on tf-fMRI data to identify ICNs anchored in regions known for articulatory, phonological, and semantic processes in healthy male and female controls (HCs). We then evaluated the reproducibility of these ICNs in an independent cohort of HCs, and recapitulated their functional relevance with a post hoc meta-analysis on task-based fMRI. Last, we investigated whether atrophy in these ICNs could inform the differential diagnosis of nonfluent/agrammatic, semantic, and logopenic PPA variants. The identified ICNs included a dorsal articulatory-phonological network involving inferior frontal and supramarginal regions; a ventral semantic network involving anterior middle temporal and angular gyri; a speech perception network involving superior temporal and sensorimotor regions; and a network between posterior inferior temporal and intraparietal regions likely linking visual, phonological, and attentional processes for written language. These ICNs were highly reproducible across independent groups and revealed areas consistent with those emerging from task-based meta-analysis. By comparing ICNs' spatial distribution in HCs with patients' atrophy patterns, we identified ICNs associated with each PPA variant. Our findings demonstrate the potential use of tf-fMRI to investigate the functional status of language networks in patients for whom activation studies can be methodologically challenging.

Key words: functional connectivity; language networks; primary progressive aphasia; reproducibility; resting-state connectivity

Significance Statement

We showed that a single, short, task-free fMRI acquisition is able to identify four reproducible and relatively segregated intrinsic left-dominant networks associated with articulatory, phonological, semantic, and multimodal orthography-to-phonology processes, in HCs. We also showed that these intrinsic networks relate to syndrome-specific atrophy patterns in primary progressive aphasia. Collectively, our results support the application of task-free fMRI in future research to study functionality of language circuits in patients for whom tasked-based activation studies might be methodologically challenging.

\section{Introduction}

Language requires the simultaneous interplay of several networks involved in the multiple processes necessary to understand and

Received June 19, 2019; revised Nov. 6, 2019; accepted Dec. 1, 2019.

Author contributions: M.L.G.-T. designed research; G.B. performed research; G.B., V.B., and M.L.M. analyzed data; M.H., M.L., Z.M., and J.D. contributed unpublished reagents/analytic tools; V.B., M.H., W.S., Z.M., J.D., B.L.M., N.D., S.M.B., W.W.S., M.L.M., and M.L.G.-T., and G.B. wrote the paper.

The study was supported by Grants from the National Institutes of Health (NINDS R01NS050915, NIDCD K24DC015544, NIDCD R03DC013403, NIDCD F32DC009145, NIDCD R01DC016291, NIA U01AG052943, NIA P50AG023501, NIA P01AG019724, NIA R01AG038791, NINDS U54NS092089; Alzheimer's Disease Research Center of California (03-75271 DHS/ADP/ARCC); Larry L. Hillblom Foundation; John Douglas French Alzheimer's Foundation; produce language, including articulatory, phonological, and semantic functions (Mesulam, 1990; Binder et al., 1997; Fedorenko and Thompson-Schill, 2014). Decades of neuroimaging research have identified regions involved in different language processes (Friederici and Gierhan, 2013), with crucial contributions from task-based functional magnetic resonance imaging studies (fMRI;

Koret Family Foundation; Consortium for Frontotemporal Dementia Research; and McBean Family Foundation. We thank the patients and their families for the time and effort they dedicated to the research.

The authors declare no competing financial interests.

Correspondence should be addressed to Giovanni Battistella at Giovanni.battistella@ucsf.edu.

https://doi.org/10.1523/JNEUROSCI.1485-19.2019

Copyright $\odot 2020$ the authors 
Price, 2012). Studies involving patients with neurological disorders have also provided essential evidence by associating structural abnormalities with specific acquired language deficits (Schwartz et al., 2012; Gorno-Tempini and Miller, 2013; Butler et al., 2014; Dronkers et al., 2017). However, combining fMRI and neuropsychological approaches has posed several significant challenges. For instance, the interpretation of fMRI activations in the context of impaired cognitive performance is unclear (Price et al., 2006a). Furthermore, individuals with aphasia are often too impaired to perform the language tasks of interest in the scanner. The introduction of task-free fMRI (tf-fMRI; Raichle, 2006) might provide a crucial alternative to investigate functional connectivity of language networks in individuals with aphasia. This technique can identify multiple functional networks defined by their intrinsic connectivity in a single MRI acquisition session and does not require participants to perform any cognitive task. To date, a number of studies have successfully used tf-fMRI to delineate reproducible networks associated with specific brain functions such as visual, sensorimotor, and executive processes, while fewer have investigated language processes (Damoiseaux et al., 2006; Fox and Raichle, 2007; Shehzad et al., 2009; Smith et al., 2009; Van Dijk et al., 2010). Whole-brain seed-based correlation analyses on tf-fMRI data serve as a robust approach to identify language intrinsic connectivity networks (ICNs), particularly as there are strong a priori hypotheses about the location of the seed regions (Bonilha et al., 2006; Mechelli et al., 2007; MacSweeney et al., 2009; Simmons et al., 2010; Buchsbaum et al., 2011). tf-fMRI studies of language processing have mainly investigated functional connectivity of the speech production and comprehension networks (Turken and Dronkers, 2011; Tomasi and Volkow, 2012; Hurley et al., 2015), but have not simultaneously characterized, within the same study, ICNs associated with other cognitive processes essential for language.

The present study aims to identify reliable ICNs anchored to brain regions crucial for language processes and to assess their relationship to atrophy patterns characteristic of the clinical variants of primary progressive aphasia (PPA). We first assessed whether whole-brain seed-based connectivity analysis on tf-fMRI could identify reproducible and spatially consistent language ICNs in healthy controls (HCs). To this end, we analyzed data from two independent cohorts of HCs. We hypothesized the existence of segregated, primarly left hemispheric, ICNs that would be reproducible across independent datasets. We then assessed whether the correlation between these ICNs and the atrophy patterns in PPA could identify the ICN/ICNs most representative of each clinical variant. To do so, we evaluated the similarity of each patient's atrophy pattern relative to the four tf-fMRI ICNs. To further examine the functional relevance of the ICNs anchored to our a priori seeds, we performed a post hoc analysis based on the overlap between ICNs and neurosynth-derived task-based metaanalytic maps. PPA is an ideal syndrome to demonstrate the application of the identified ICNs to disease, since each of the three variants demonstrates selective impairment in language processes supported by the HC-derived ICNs. We hypothesize that language ICNs would be reliable and reproducible, that they would overlap with atrophy maps in PPA variants, and that they would correspond to functional networks derived from taskbased activation maps.

\section{Materials and Methods Participants}

Sixty-four healthy volunteers were recruited at the Memory and Aging Center (MAC) at the University of California, San Francisco (UCSF). All
Table 1. Demographic, clinical, and neuropsychological characteristics of patients and controls

\begin{tabular}{|c|c|c|c|c|}
\hline & HCscore & IvPPA score & svPPA score & nfvPPA score \\
\hline \multicolumn{5}{|l|}{ Demographics } \\
\hline Age & $66.58(5.39)$ & $64.05(7.69)$ & $63.58(6.81)^{*}$ & $67.81(7.71)$ \\
\hline Gender (M/F) & $24 \mathrm{M} / 40 \mathrm{~F}$ & $19 / 25$ & $36 / 30$ & $15 / 36$ \\
\hline Education & $17.56(2.06)$ & $16.65(2.74)$ & $16.33(2.89)^{*}$ & $16.41(3.32)^{*}$ \\
\hline \multicolumn{5}{|l|}{ Clinical } \\
\hline MMSE (30) & $29.53(0.78)$ & $21.6(5.52)^{*}$ & $22.58(6.54)^{*}$ & $24.69(5.24)^{*}$ \\
\hline CDRTot & $0.01(0.07)$ & $0.54(0.24)^{*}$ & $0.65(0.29)^{*}$ & $0.46(0.3)^{*}$ \\
\hline CDR box score & $0.02(0.1)$ & $2.94(1.52)^{*}$ & $3.66(2.12)^{*}$ & $1.98(1.39)^{*}$ \\
\hline Onset & $\mathrm{NaN}$ & $59.07(7.29)$ & $58.94(6.84)$ & $64.11(6.99)$ \\
\hline \multicolumn{5}{|l|}{ Language Production† } \\
\hline Boston Naming Test (15) & $13.8(1.4)$ & $9.35(3.95)^{*}$ & $3.95(3.29)^{*}$ & $11.65(3.3)^{*}$ \\
\hline Phonemic fluency (30) & 15.1( & 8.44( & $7.07(4.24)^{*}$ & $5.6(4.79)^{*}$ \\
\hline Semantic fluency (40) & $21.67(5.68)$ & $8.12(4.74)^{*}$ & $7.52(4.87)^{*}$ & $10.0(6.56)^{*}$ \\
\hline WAB fluency (10) & $10(0)$ & $8.36(1.09)^{*}$ & $8.71(1.04)^{*}$ & $6.31(2.74)^{*}$ \\
\hline AOS rating (7) & $0.0(0.0)$ & $0.34(1.16)$ & $0.09(0.47)$ & $2.54(1.93)^{*}$ \\
\hline Dysarthria rating (7) & $0.0(0.0)$ & $0.0(0.0)$ & $0.09(0.47)$ & $2.17(2.53)^{*}$ \\
\hline WAB repetition (100) & $99.5(0.9)$ & $73.54(11.01)^{*}$ & $87.81(13.02)^{*}$ & $81.11(19.38)^{*}$ \\
\hline \multicolumn{5}{|l|}{ Language comprehensiont } \\
\hline PPVT (16) & $15.38(1.06)$ & $13.51(2.33)^{*}$ & $7.67(4.07)^{*}$ & $13.88(2.44)^{*}$ \\
\hline $\begin{array}{l}\text { WAB sequential commands } \\
(80)\end{array}$ & $80(0)$ & $65.53(11.73)^{*}$ & $69.45(16.43)^{*}$ & $69.69(11.82)^{*}$ \\
\hline $\begin{array}{l}\text { Pyramids and palm trees, } \\
\text { pictures (52) }\end{array}$ & $49.5(4.65)$ & $48.22(3.87)$ & $40.4(7.67)^{*}$ & $47.48(5.11)^{*}$ \\
\hline \multicolumn{5}{|l|}{ Visuospatial functiont } \\
\hline Benson copy (17) & $15.73(0.88)$ & $14.76(2.17)^{*}$ & $15.46(1.18)$ & $14.33(2.1)^{*}$ \\
\hline \multicolumn{5}{|l|}{ Visual memoryt } \\
\hline Benson delayed re & $12.78(2.3)$ & $6.24(3.79)^{*}$ & $7.42(4.5)^{*}$ & $9.58(3.65)^{*}$ \\
\hline \multicolumn{5}{|l|}{ Verbal memory $\dagger$} \\
\hline CVLT-MS Trial 1-4 (36) & $29.57(3.09)$ & $12.49(6.89)^{*}$ & $14.92(7.18)^{*}$ & $21.85(6.68)^{*}$ \\
\hline CVLT-MS 30 Sec delay (9) & $7.92(1.18)$ & $2.78(2.41)^{*}$ & $2.37(2.45)^{*}$ & $5.94(2.42)^{*}$ \\
\hline CVLT-MS 10 min delay (9) & $7.5(1.3)$ & $2.12(2.66)^{*}$ & $1.59(2.18)^{*}$ & $5.52(2.48)^{*}$ \\
\hline \multicolumn{5}{|l|}{ Executive functiont } \\
\hline Digit span backward (8) & $5.5(1.32)$ & $3.15(0.99)^{*}$ & $4.57(1.23)^{*}$ & $3.28(1.36)^{*}$ \\
\hline $\begin{array}{l}\text { Modified trails (lines per } \\
\text { minute) }\end{array}$ & $35.34(13.44)$ & $9.73(8.87)^{*}$ & $20.86(12.66)^{*}$ & $12.11(10.49)^{*}$ \\
\hline Calculation (5) & $4.65(0.58)$ & $3.39(1.12)^{*}$ & $4.46(0.68)$ & $4.22(0.98)^{*}$ \\
\hline
\end{tabular}

Values are mean (SD).

*Significantly impaired relative to controls, $p<0.05$

†Scores were not available for all the participants involved in the study, so we report mean and SD of the subset of participants included. WAB scores for HC are taken from Gorno-Tempini et al. (2004). WAB, Western Aphasia Battery; AOS, Apraxia of Speech; PPVT, Peabody Picture Vocabulary Test; CDR, Clinical Dementia Rating; CVLT-MS, California Verbal Learning Test-Mental Status.

controls denied a history of neurological and psychiatric disorders, and achieved normal scores on the Mini-Mental State Examination.

We recruited 161 patients with a probable diagnosis of primary progressive aphasia according to clinical consensus criteria (Gorno-Tempini et al., 2011). Among the patients, 51 were diagnosed with non-fluent variant PPA (nfvPPA), 44 with logopenic PPA (lvPPA), and 66 with semantic PPA, (svPPA). All patients received comprehensive multidisciplinary evaluations, including neurological history and examination, neuropsychological testing, and neuroimaging, as previously described (Gorno-Tempini et al., 2004). Patients had a Clinical Dementia Rating (CDR) score $\leq 1$. We provide demographic, clinical, and neuropsychological characteristics for patients and HCs in Table 1, which shows the expected clinical profile of patients with the three PPA variants. According to the literature, nfvPPA patients are characterized by motor speech impairment consistent with apraxia of speech, often with dysarthria, and/or agrammatism; the left inferior frontal gyrus is considered as the syndrome-specific epicenter, and associated with atropy in the insula, premotor regions, SMA, and striatum (Grossman et al., 1996). SvPPA patients are clinically characterized by poor performance on confrontation naming, single word comprehension, and surface dyslexia; the left anterior temporal lobe is considered as the syndrome-specific epicenter, with atrophy progressing along temporal regions (Hodges et al., 1992). LvPPA patients are clini- 
cally characterized by word finding difficulties, along with sentence repetition and phonological short-term memory deficits; cortical atrophy is typically located in posterior superior and middle temporal gyri as well as the inferior parietal lobule (Henry and Gorno-Tempini, 2010).

Participants provided written informed consent, which was approved by the Internal Review Board at the University of California, San Francisco.

The following sections refer to the description of tf-fMRI data in the two independent groups of HCs used to address the first and third aims of the study, and one section describes the procedure developed at UCSF to quantify single subject atrophy patterns in patients (Aim 2).

\section{MRI protocol}

The neuroimaging protocol for all 64 controls included a high-resolution MRI structural scan for intersubject registration, as well as an echoplanar imaging (EPI) scan to study task-free functional connectivity. Participants were instructed to remain still and keep their eyes closed without falling asleep during the acquisition of task-free (tf-fMRI) data. Participants were scanned with a Siemens 3-Tesla Trio scanner using a body transmit coil and an 8-channel receive head coil. A T1-weighted 3D magnetization prepared rapid acquisition gradient echo (MPRAGE) was acquired with 160 sagittal slices, TE/TR/TI $=2.98 / 2300 / 900 \mathrm{~ms}$, flip angle $=9^{\circ}$, isotropic voxel with size of $1 \mathrm{~mm}$, field-of-view $=256 \times 256$ $\mathrm{mm}$, matrix $=256 \times 256$. For tf-fMRI, we acquired $240 \mathrm{~T} 2^{\star}$-weighted EPI volumes in interleaved order consisting in $36 \mathrm{AC} / \mathrm{PC}$-aligned axial slices with the following parameters: TR/TE $=2000 / 27 \mathrm{~ms}$, flip angle $=$ $80^{\circ}$, slice thickness $=3 \mathrm{~mm}$ with $0.6 \mathrm{~mm}$ gap, field-of-view $=230 \times 230$ $\mathrm{mm}$, matrix $=92 \times 92$ in plane resolution. The total acquisition time of the tf-fMRI was 8 min and $6 \mathrm{~s}$, including the initial three "dummy scans".

\section{Preprocessing of tf-fMRI}

The analysis of functional data was performed using an in-house pipeline that makes use of tools available in FSL v5 (http://fsl.fmrib.ox.ac.uk/fsl/ fslwiki/), AFNI (http://afni.nimh.nih.gov/afni/), and Numpy (Python v2.7.3). The first five volumes of the acquisition were discarded to allow T1 equilibrium to be established. Functional images first were corrected for slice timing and checked for excessive motion after the realignment of the volumes to the mean functional image. None of the participants exceeded a maximum of $2 \mathrm{~mm}$ for relative head motion, a maximum of $2^{\circ}$ for relative rotation, and a maximum of $10 \%$ of the total frames with motion spikes, calculated as relative motion $>1 \mathrm{~mm}$. The mean functional image then was coregistered with the 3D MPRAGE using a rigid body transformation. The structural image was then normalized to the MNI space, and the transformation matrix subsequently applied to the tf-fMRI data that were finally spatially smoothed with a Gaussian kernel of $6 \mathrm{~mm}$ FWHM. CSF and white matter tissue probability maps, calculated with the FSL FEAT tool, were then used to compute the mean time-series used as regressors. Functional data were then bandpass filtered $(0.008 \mathrm{~Hz}<f<0.15 \mathrm{~Hz})$ and the nuisance variables were regressed out from the data, which included the six motion parameters, CSF and white matter time series as well as the first derivative and quadratic terms, as suggested in (Satterthwaite et al., 2013). Spectral filtering and nuisance regression were combined into a single step using AFNI tools (Hallquist et al., 2013).

\section{Functional definition of language networks: seed ROI definition}

The seed ROIs used to identify language-related functional connectivity networks were defined as $8 \times 8 \times 8 \mathrm{~mm}^{3}$ boxes, derived by combining evidence from neuroimaging and cognitive studies in PPA, neuropsychological studies on other patient populations, and task-based fMRI studies in HCs (Fig. 1A). An extensive description of the rationale behind the choice of three of the four seeds (left opercular IFG, anterior MTG, and posterior ITG) used in the current study is presented in (Battistella et al., 2019). Here we present a brief summary as well as the motivation for selection of the fourth seed.

The first seed, included for its relevance in articulatory-phonological processes, was defined in the left posterior, opercular part of the IFG (opIFG) at the activation peak (MNI coordinates: $x=-50, y=8, z=23$ )

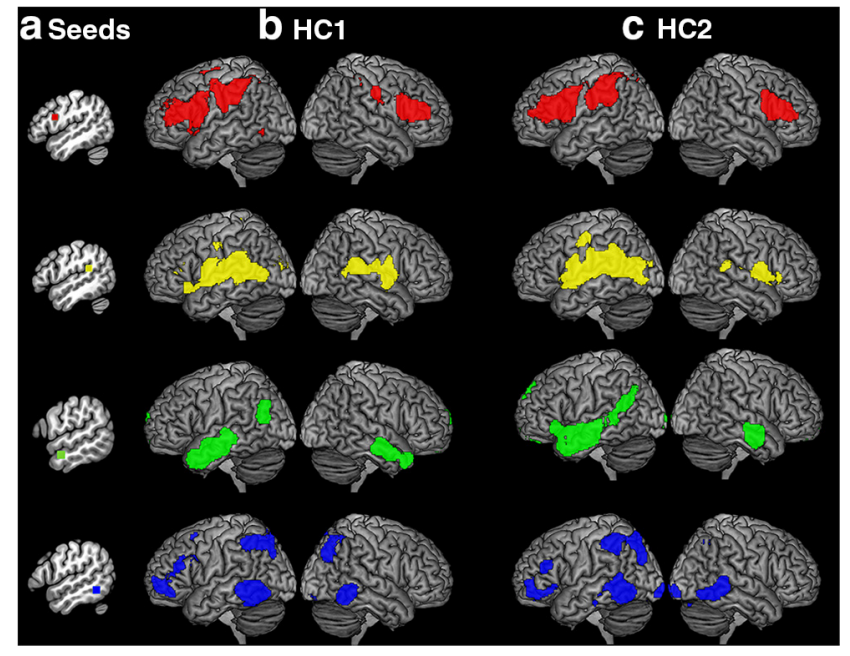

Figure 1. Identification and reproducibility of the intrinsic language networks. The seeds used to build the networks are shown in a sagittal view of a standard brain in the MNI space $(\boldsymbol{a})$. The resulting networks are displayed on a three-dimensional rendering of the Montreal Neurological Institute standard brain at $p<5 \times 10^{-5}$ after FWE correction for multiple comparisons at peak level over the whole brain and $k>180$ for cluster extent for $\mathrm{HC}(\boldsymbol{b})$ and $\mathrm{HC2}$ (c). The significant voxels of each network were binarized and shown in blue (network seeded from the posterior ITG, pITG), yellow (network seeded from the Superior parietal temporal region, Spt), red (network seeded from the opercular IFG, opIFG), and green (network seeded from the anterior MTG, aMTG).

identified in a previous study by contrasting a phonemic fluency task against semantic and syntactic fluency tasks (Heim et al., 2008). As described by Battistella et al. (2019), many functional peaks from studies of verbal fluency and articulation previously identified in the fMRI literature fall in our $8 \mathrm{~mm}$ box. This region is atrophic in the nfvPPA and frequently lesioned in cases of post-stroke Broca's aphasia (Hillis et al., 2004). The second seed (MNI coordinates: $x=-51, y=-42, z=21$ ) was defined in a portion of the planum temporale/parietal operculum and is a subportion of area Tpt (Galaburda and Sanides, 1980). The coordinates of this seed have been chosen from a phonological working memory task (conjunction of encoding and delay contrasts) in a group of 105 healthy participants (Buchsbaum et al., 2011). The language model developed by Hickok and Poeppel (2004) defines this region as superior parietal temporal (Spt) and represents a key region of the dorsal stream involved in speech perception (Milner and Goodale, 1995; Humphries et al., 2005). It was initially identified in an anatomically unconstrained analysis that specified regions showing both auditory (speech responsive) and motor (responsive during covert speech production) response properties (Hickok and Poeppel, 2004; Buchsbaum et al., 2011). Morphometric studies in PPA associate atrophy in this region at the temporoparietal junction with the lvPPA (Gorno-Tempini et al., 2004).

A third seed, potentially relevant for semantic processing, was defined in the left anterior middle temporal gyrus (aMTG) at the activation peak (MNI coordinates: $x=-60, y=-6, z=-18$ ) identified in a previous study by contrasting a semantic association task on pairs of famous faces against a perceptual matching task related to pairs of unknown faces (Gesierich et al., 2012). As described by Battistella et al. (2019), many functional peaks from studies of semantic processing previously identified in the fMRI literature fall in our $8 \mathrm{~mm}$ box. This region is also damaged in the svPPA.

The fourth seed was defined in the left posterior ITG (pITG) at the peak (MNI coordinates: $x=-54, y=-52, z=-10$ ) of a cluster that showed greater activation in controls than in svPPA patients when contrasting a semantic judgment task against a visual judgment task (Mummery et al., 1999). This seed is considered a multimodal language region where visual and nonvisual components of reading converge (Büchel et al., 1998). A task-based fMRI study directly associates activity in this region with performance on an auditory spelling task (Booth et al., 2003). This area is considered an important gateway hub involved in linking visual asso- 
ciation areas with phonological and linguistic processes in superior temporal and inferior parietal areas (Price, 2000; Bolger et al., 2008; Kuperberg et al., 2008; Price, 2012). The pITG is often atrophied in lvPPA, which is characterized by phonological deficits and phonological dyslexia. As described by Battistella et al. (2019), this area and the associated functional network have been associated to orthography-tophonology conversion based on the significant correlation of the pseudoword reading score with the pITG-to-inferior parietal cortex functional connectivity in svPPA.

\section{Seed-based functional connectivity analysis}

Single-subject correlation maps were generated by calculating the correlation coefficient between the average BOLD signal time course from the seed ROIs and the time course from all other voxels of the brain in each subject's native space. The selected seed regions of interest were normalized from the MNI standard space back to the subjects' native space using the previously estimated normalization parameters. We carefully checked the quality of the spatial normalization and location of the warped seeds in the native space to ensure the correct application of the transformations and consistent anatomical localization across participants. For each subject, the average time series from the seed was extracted and used to compute the temporal correlation against all the other voxels in the brain to create an $r$-Pearson correlation map of each voxel's connectivity strength to the seed region of interest. Correlation maps were converted to $z$-scores by Fisher's r-to- $z$ transformation and back-transformed to the MNI space to enable parametric statistical comparisons.

\section{Aim 1: characterization and reproducibility of the language ICNs in HCs}

Experimental design and statistical analysis: identification of the language networks in two groups of HCs

We divided the study sample of 64 healthy volunteers into two groups of 32 participants to have an independent dataset to study the reproducibility of the tf-fMRI ICNs. The first group (hereafter called HC1) included 13 men and 19 females (mean age \pm SD: $68.8 \pm 5.3$ years, 4 left-handed). The second group (HC2) was age- and gender-matched with $\mathrm{HC} 1$ and included 11 men and 21 females (69.8 \pm 5.3 years, 5 left-handed).

Identification of language networks was performed on the correlation maps converted to $z$-scores using a one-sample $t$ test for each group with gender and age as covariates of no interest. Statistical thresholds on the resulting group-level connectivity maps were applied at $p<5 \times 10^{-5}$ after peak-level familywise error (FWE) correction for multiple comparisons over the whole brain and $k>180$ for cluster extent (greater than the minimum number of voxels expected per cluster).

We then tested the reproducibility of the ICNs between the two groups of HCs by means of the Dice coefficient (DC) based on the spatial overlap of the cortical labels of the Harvard-Oxford atlas (https://fsl.fmrib.ox.ac. uk/fsl/fslwiki/Atlases).

\section{Characterization of the networks in terms of spatial overlap and lateralization}

We performed a conjunction analysis of the two groups to study the relative spatial distribution of the four networks and their reciprocal overlap. Statistical threshold and covariates of no interest to extract those maps were the same as the ones used to identify the networks separately in each group.

Hemispheric dominance of the extracted networks was tested using the laterality index (LI), as implemented in the AveLI toolbox (Matsuo et al., 2012). An LI coefficient of 1 represents a complete left lateralization of the networks, whereas -1 represents a full right lateralization. Subsequent analyses were performed on the ICNs derived from the conjunction analysis.

\section{Aim 2: associate the ICNs identified in controls with the atrophy patterns of PPA patients \\ Define the atrophy pattern of patients through the calculation of $w$-maps}

The w-map is a voxel-based measure that reveals whole-brain atrophy patterns of an individual patient compared with a large number of HCs.
$\mathrm{W}$-maps rely on the calculation of the $w$-score from preprocessed T1weighted images.

Preprocessing of T1-weighted images. Structural T1-weighted images were preprocessed using SPM12 (https://www.fil.ion.ucl.ac.uk/spm/ software/spm12/). Preprocessing involved bias-correction, segmentation into tissue probability maps, and spatial normalization using a single generative model with the standard SPM12 parameters. To optimize intersubject registration, each of the patients' gray matter probability maps were warped to a template derived from 300 confirmed neurologically healthy older adults [ages $44-86$, mean $(\mathrm{M}) \pm$ SD: $67.2 \pm$ $7.3 ; 113$ males, 186 females] scanned with one of three magnet strengths $(1.5 \mathrm{~T}, 3 \mathrm{~T}, 4 \mathrm{~T})$, using affine and nonlinear transformations using the diffeomorphic anatomical registration through exponentiated lie algebra (DARTEL) toolbox (Ashburner, 2007). Spatially normalized, segmented, and modulated gray matter images were smoothed using an $8 \mathrm{~mm}$ FWHM isotropic Gaussian kernel.

Calculation of the $w$-maps. Smoothed modulated and normalized gray matter maps (smwc1) of 534 confirmed neurologically HCs from the UCSF MAC Hilblom Cohort (age range 44-99 years, M \pm SD: $68.7 \pm$ $9.1 ; 220$ male/302 female) were regressed against age, sex, total intracranial volume, and magnet strength. The $\beta$ values of this regression were then used to calculate the predicted smwcl values for each patient. The actual patient's smwclvalues were then subtracted from the predicted values and divided by the SD of the residuals in the control group to get the individual patient's w-map. Obtained $w$-scores (e.g., applied in neurodegeneration by La Joie et al., 2012; Ossenkoppele et al., 2015) are interpreted like $z$-scores, with mean $=0 / \mathrm{SD}=1$. Negative $w$-scores represent below-average volume. W-maps were thresholded at scores $\leq-2.50$ to create the atrophy maps of patients. These analyses were performed using the in-house Brainsight system, developed at UCSF by Katherine P. Rankin, Cosmo Mielke, and Paul Sukhanov, and powered by the VLSM script written by Stephen M. Wilson, with funding from the Rainwater Charitable Foundation and the UCSF Chancellor's Fund for Precision Medicine.

\section{Identification of the ICN most representative of the atrophy distribution of each PPA variant}

We calculated the percentage of overlap of the atrophy map of each patient with each of the $4 \mathrm{ICNs}$, resulting in a $161 \times 4$ matrix. We then examined similarity relationships of the correlation of each subject' atrophy map with the 4 ICNs (resulting in a $161 \times 161$ similarity matrix). To this end, we used custom Python scripts relying on Numpy (http:// www.numpy.org/), and Scipy (http://www.scipy.org/scipylib). Finally, for each patient, we adopted a "winner takes all" approach to identify the ICN with the highest overlap with the corresponding w-map. Based on that we determined which ICN aligned the most with the atrophy distribution of each PPA variant.

\section{Aim 3: meta-analysis on task-based fMRI studies}

The Neurosynth toolbox (Yarkoni et al., 2011) was used to perform a post hoc meta-analysis on previous task-based language-related fMRI studies. This post hoc analysis was performed to visually characterize the overall similarity between the ICNs anchored to our a priori ROIs and functional maps obtained from previous language activation studies. Neurosynth is an open-source resource that performs automated meta-analyses. The full text of all articles in the database is parsed, and each article is "tagged" with a set of terms that occur at a high frequency in that article. A metaanalysis is then performed by automatically comparing the coordinates reported for studies with and without the term of interest (key words). In the present study, we performed the meta-analysis using the following keywords: speech production (removing speech perception), speech perception (removing speech production), semantic memory, and orthography, which were consistent with the hypothesized role of each intrinsic $\mathrm{ROI} / \mathrm{tf}$-fMRI networks. Because of the post hoc nature of this analysis and the intrinsic limitation of Neurosynth related to the impossibility to control for specific experimental factors in each of the studies included in the meta-analysis, the resulting maps were only visually inspected and no formal quantitative overlap analysis was conducted. 
Table 2. Significant clusters of the four intrinsic language networks in HCs

\begin{tabular}{|c|c|c|c|c|c|}
\hline & \multicolumn{3}{|c|}{ Coordinates } & \multirow[b]{2}{*}{ Tscore } & \multirow[b]{2}{*}{ Cluster size } \\
\hline & $x$ & $y$ & $z$ & & \\
\hline \multicolumn{6}{|l|}{ Left opIFG-seeded network } \\
\hline Left opIFG & -50 & 10 & 22 & 38.6 & 6068 \\
\hline Left SMG & -55 & -34 & 34 & 12.3 & * \\
\hline Left superior IPL (BA 40) & -56 & -36 & 44 & 14.2 & * \\
\hline Left A30:F53triangular IFG & -44 & 38 & 16 & 13.8 & * \\
\hline Right opIFG & 52 & 32 & 18 & 13.9 & 1369 \\
\hline SMA & -2 & 10 & 46 & 13.3 & 688 \\
\hline \multicolumn{6}{|l|}{ Left Spt-seeded network } \\
\hline Left posterior superior temporal (Spt area) & -50 & -42 & 20 & 33.1 & 5485 \\
\hline Left middle temporal lobule & -50 & -54 & 12 & 14.2 & * \\
\hline left rolandic operculum & -54 & -4 & 8 & 13.9 & * \\
\hline Left postcentral gyrus & -42 & -14 & 38 & 10.5 & 206 \\
\hline Middle cingulate cortex & -2 & 12 & 38 & 11 & 190 \\
\hline Right Heschl gyrus & 48 & -12 & 10 & 11.6 & 469 \\
\hline Right rolandic operculum & 60 & 0 & 12 & 11.4 & $*$ \\
\hline Right superior temporal & 56 & -42 & 18 & 11.2 & 252 \\
\hline Right precuneus & 4 & -46 & 50 & 10.7 & 184 \\
\hline \multicolumn{6}{|l|}{ Left aMTG-seeded network } \\
\hline Left anterior MTG & -60 & -6 & -18 & 42.7 & 1839 \\
\hline Left angular gyrus & -46 & -62 & 34 & 10.7 & 425 \\
\hline Medial prefrontal cortex & 0 & 60 & -8 & 14 & 1399 \\
\hline Posterior cingulate cortex & -3 & -49 & 33 & 11 & $*$ \\
\hline Right anterior MTG & 60 & -10 & -18 & 13.4 & 512 \\
\hline \multicolumn{6}{|l|}{ Left pITG-seeded network } \\
\hline Left posterior ITG & -54 & -52 & -10 & 36.8 & 1675 \\
\hline Left middle temporal gyrus & -62 & -42 & 0 & 11.4 & $*$ \\
\hline Left Intraparietal sulcus (BA 40) & -48 & -44 & 52 & 14.9 & 2606 \\
\hline Left superior parietal lobule & -28 & -70 & 46 & 14.9 & * \\
\hline Left middle occipital & -28 & -72 & 38 & 14 & * \\
\hline Left triangular IFG & -48 & 32 & 20 & 13.7 & 336 \\
\hline Left orbital IFG & -46 & 42 & -12 & 11.4 & 471 \\
\hline Left Crus I & -6 & -76 & -20 & 11.3 & 206 \\
\hline Right posterior ITG & 60 & -52 & -8 & 15.4 & 658 \\
\hline Right superior parietal & 32 & -64 & 54 & 11.3 & 498 \\
\hline
\end{tabular}

*Belong to the same cluster.

\section{Results}

Aim 1: characterization and reproducibility of the language ICNs in HCs

Identification of the language networks in the two groups of HCs The language networks identified in the two independent groups of HCs showed a similar spatial distribution, with voxels from HC1 highly overlapping with those in HC2 (Fig. 1). The DCs showed that the aMTG- and PITG- seeded ICNs were the most overlapping networks across the two groups of $\mathrm{HCs}$ with a DC of 0.83 . The ICN-seeded network derived from the opIFG showed a DC $=0.75$, whereas the Spt-seeded a DC $=$ 0.74 .

The network resulting from the left opIFG seed included areas in the bilateral opercular and triangular part of the IFG, left middle frontal gyrus, bilateral SMG and IPS, left putamen, left pre SMA, and left ITG. The network from the left Spt seed included areas in the bilateral planum temporale, left precentral and SMG, as well as in the left postcentral gyrus, STG, anterior cingulate cortex, and right fusiform gyrus. The network from the left aMTG seed included instead areas in the bilateral MTG, temporal pole, ITG, precuneus, hippocampus, and parahippocampal gyrus. Additional significant regions were located in the left angular gyrus, anterior insula, orbital IFG, anterior and posterior cingulate cortices, as well as in the right middle occipital, and orbital medial frontal gyrus. Finally, the network from the left pITG seed included areas in the bilateral ITG, left intraparietal sulcus, and bilateral triangular IFG. Figure 1 illustrates these results.

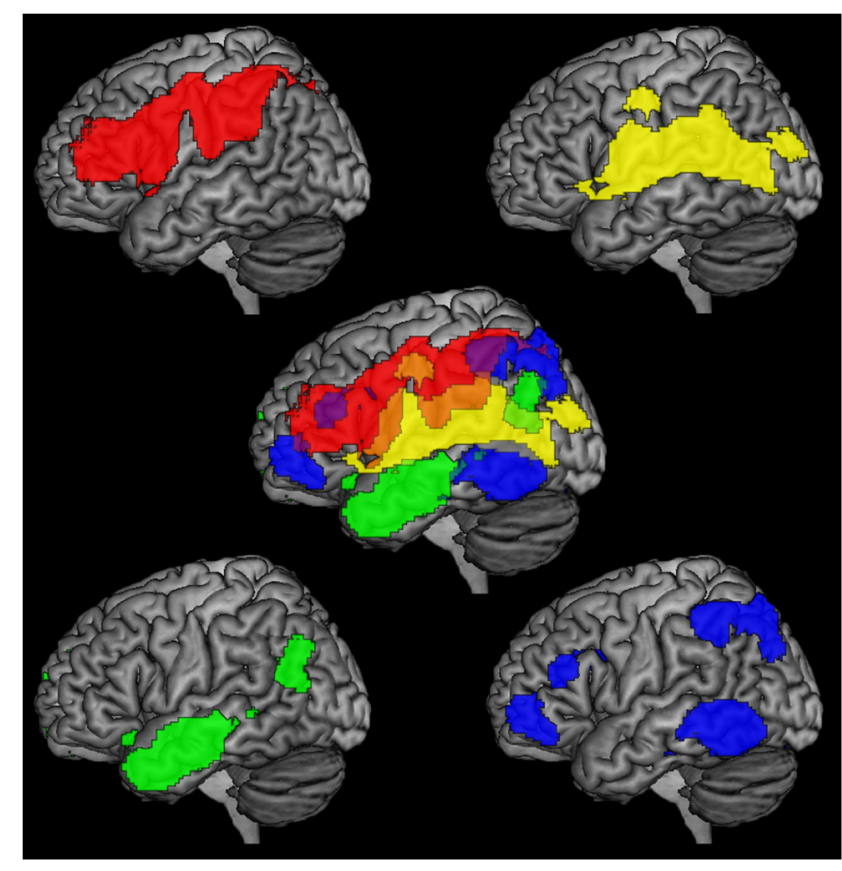

Figure 2. Spatial distribution of the four task-free networks. Rendered left-hemisphere lateral views of the four ICNs identified through a statistical conjunction of the two groups. Maps were thresholded at the same statistical threshold used to identify the networks for each group separately, binarized and colored using the same color scheme in Figure 1 (blue for the network seeded from the pITG, yellow for the network seeded from the Spt, red for the network seeded from the opIFG, and green for the one seeded from the aMTG). The render at the center of the figure shows that the four networks cover almost the entire left hemisphere with a small degree of overlap.

These findings showed high reproducibility of the reconstructed language networks across two independent datasets of healthy older adults.

\section{Characterization of the networks in terms of spatial overlap and lateralization}

Table 2 lists peak coordinates of significant clusters of the conjunction analysis. The four networks extensively covered the left hemisphere and were mostly spatially distinct from one another (Fig. 2). The DC showed, in fact, a value equal or $<0.2$, corresponding to a very low degree of overlap. Motor and sensorimotor regions of the network originating from the Spt overlapped with the same regions of the opIFG-seeded network, while inferior and middle frontal clusters, as well as inferior parietal regions overlapped between the opIFG and the pITG networks.

The LI analysis showed a strong lateralization in the left hemisphere of the networks seeded from the pITG, Spt, and opIFG ( $\mathrm{LI}=0.50,0.62,0.58$ respectively). The network originating from the aMTG was instead less left lateralized than the other intrinsic networks, with a $\mathrm{LI}=0.37$.

Aim 2: associate the ICNs identified in controls with the atrophy patterns of PPA patients

Identification of the ICN most representative of each PPA variant The similarity matrix shows the homogeneity of the 3 PPA groups in terms of their similarity coefficients measured using the overlap between the 4 ICNs derived from HCs and each patient's atrophy map (Fig. 3). The nfvPPA and svPPA were highly homogenous in term of similarity coefficients. The lvPPA patients were instead less so, in line with the high intersubject variability of the patients affected by this syndrome (Louwersheimer et al., 
2016). The winner takes all approach showed that $80 \%$ of the nfvPPA atrophy maps mostly overlapped with the ICN derived using the opIFG seed; $92 \%$ of svPPA atrophy maps were associated with the ICN seeded from the aMTG. We did not find a unique ICN representative of the lvPPA patients, whom atrophy patterns were instead associated with a combination of the aMTG- (in $43 \%$ of the patients), Spt- (23\% of the patients), and pITG- (25\% of patients) seeded networks.

\section{Aim 3: meta-analysis on task-based fMRI studies}

The task-based fMRI meta-analysis obtained with 128 studies sorted using the keyword "speech production" and removing the effect of "speech perception" showed a map covering activations in the bilateral opIFG, precentral gyrus, and STG. Additional clusters were located in the left postcentral gyrus and basal ganglia. This pattern shows similarities with the tf-fMRI network originated from the seed in the opIFG. The two differed mainly in the activation of the STG in the taskbased map that was instead absent in the opIFG-seeded ICN. We ascribe the presence of the temporal activation to the intrinsic limitation of the automatic meta-analytic approach adopted. The fMRI experiments included in the analyses, even if associated with speech production rather than perception, might have failed to appropriately control for auditory feedbacks and primary auditory activations. The 150 studies that entered the meta-analysis using the term speech perception showed activations in the bilateral STG and MTG as well as in the left precentral gyrus, resembling the spatial distribution of the tf-fMRI network using the Spt seed. The 204 studies identified in the Neurosynth meta-analysis with the keyword "semantic memory" showed activations in medial regions in the PCC, precuneus, orbitofrontal regions, as well as in the inferior, middle temporal, and triangular inferior frontal gyri. This pattern of activation resembles, among the four tffMRI networks, the network created using the seed from the aMTG. Finally, the meta-analysis with the 132 studies using the keyword "orthography" showed a statistical map that covered regions in the left inferior frontal gyrus, left inferior and superior parietal lobule, inferior and middle temporal gyri, and SMA. This is consistent with the tf-fMRI map from the p-ITG. These results are visually summarized in Figure 4.

\section{Discussion}

Using whole-brain, seed-based connectivity from tf-MRI data in HCs, this study identified four intrinsic language networks related to essential language cognitive processes (i.e., speech production and perception, semantics, and orthography); our findings are supported further by a post hoc meta-analysis of taskbased fMRI studies with Neurosynth. By replicating results with two independent cohorts of controls, we demonstrated there was a high reproducibility of the identified ICNs, confirming the existence of robust and dissociable language ICNs at rest. These networks were segregated topographically with a small degree of overlap, and extensively covered the left hemisphere. Languagerelated networks were strongly left-lateralized, with the exception of the ventral semantic ICN, which comprised medial prefrontal and posterior cingulate regions included in the default-mode network (DMN). Finally, we demonstrated a successful application of the identified ICNs by showing distinctive overlap between patterns of intrinsic connectivity in controls and atrophy distribution in PPA; these patterns were able to identify which ICN/ ICNs were most representative of each of the three variants of this syndrome. We discuss possible functional roles of these networks in specific language processes based on literature review of tf- and task-based fMRI studies, the latter being instrumental to interpret the ICNs map in relation to their functions, in controls and patients.

The ICN originating from the left opIFG seed included regions involved in cognitive processes required to produce meaningful and correctly articulated utterances (SMA, premotor, supramarginal, and anterior insula regions) and grammatical functions (prefrontal and middle frontal gyri), collectively referred to as speech production (Broca, 1861; Dronkers and Ogar, 2004; Hillis et al., 2004). Structural MRI analyses in PPA and activation $\mathrm{fMRI}$ studies in controls also confirmed these findings (Price et al., 2006b; Grossman, 2012; Price, 2012). In particular, the posterior opIFG and its structural connections are damaged in nfvPPA patients (Grossman et al., 1996), and DTI-derived neuroimaging metrics in the opIFG network were associated with the severity of motor speech and fluency impairments (Catani et al., 2013; Mandelli et al., 2014, 2016). More anterior portions of IFG and prefrontal areas, including the pars triangularis and middle frontal gyrus, instead are involved in grammatical processes 


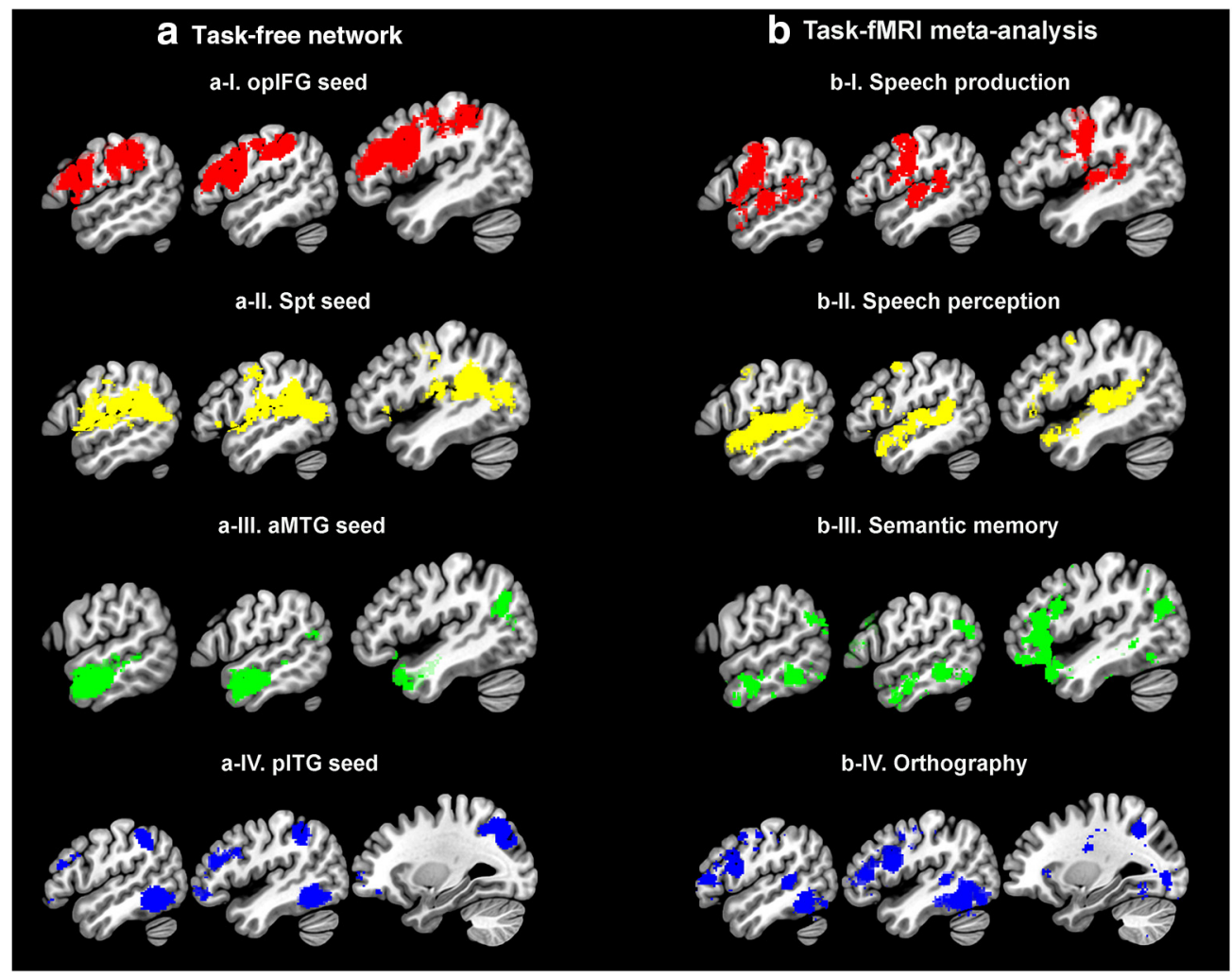

Figure 4. Task-based derived networks identified through meta-analysis. $\boldsymbol{a}$, Sagittal slices of the ICNs identified with tf-fMRI and already shown in Figure 2 . $\boldsymbol{b}$, Results of the meta-analysis on task-based fMRI studies using the following terms to create the functional maps: speech production $(\boldsymbol{b}-\boldsymbol{I})$, speech perception $(\boldsymbol{b}-\boldsymbol{I I})$, semantic memory $(\boldsymbol{b}$-III), and orthography $(\boldsymbol{b}-\boldsymbol{I V})$. The functional maps resulting from the meta-analysis using the abovementioned terms recapitulate on the cognitive functions associated to each of the tf-fMRI ICNs.

in both HCs and patients (Friederici et al., 2006; Amici et al., 2007; Wilson et al., 2011; den Ouden et al., 2019). Our results show that a short tf-fMRI acquisition is able to identify the highly predictable anatomy of the speech production network.

The role of the Spt network in sensorimotor processes for speech perception has been highlighted in recent cognitive models of language (Hickok and Poeppel, 2007). In particular, taskbased fMRI and MEG studies support the role of the Spt region in sensorimotor processes (Okada and Hickok, 2006; Pa and Hickok, 2008; Houde and Nagarajan, 2015). Brain-behavior research also has shown that this area is consistently damaged in patients with conduction aphasia and short-term phonological memory and repetition deficits (Buchsbaum et al., 2011). Notably, this network extends to posterior MTG and STG regions previously identified as portions of the classic Wernicke's area, suggesting that the network contributes to higher level lexical processes (Turken and Dronkers, 2011). This ICN also showed the greatest overlap with the network anchored to the left opIFG in frontal and motor cortices. Task-fMRI, ecoG, and EEG studies have demonstrated that these areas are essential not only for articulation and speech production, but also for auditory-motor processing of speech sounds and repetition tasks (Wilson et al., 2004; Möttönen et al., 2013; Cheung et al., 2016).

The network originating from the aMTG involved ventral temporo-angular regions consistently associated with language comprehension and semantic processing in fMRI activation studies of HCs and patients (Gorno-Tempini et al., 1998; Binder et al., 2003; Mechelli et al., 2007; Seghier et al., 2010; Gesierich et al., 2012). In addition to middle temporal and angular regions, the ICN included medial prefrontal and posterior cingulate regions that are part of the DMN. The activation of the DMN in task-free functional studies is well established and has been associated with several cognitive processes (Greicius et al., 2003), including semantics (for meta-analysis, see Binder et al., 2009). The aMTG region used as a seed to build this network consistently is damaged in svPPA patients with profound semantic memory loss (Hodges et al., 1992), and recent studies have shown decreased functional connectivity of this region to the angular gyrus in this patient population (Battistella et al., 2019; Montembeault et al., 2019). Recent models of language suggest that the angular gyrus is involved in retrieval and/or executive semantic processes (Binder and Desai, 2011; Ralph et al., 2017).

The pITG-anchored network included regions often part of the "dorsal attention" network (Corbetta et al., 2000; Maldjian et al., 2003; Fox et al., 2006). This circuit is involved in verbal and visual working memory processes necessary for reorienting attention in response to salient stimuli, which is required for cognitive processes such as reading and spelling (DeMarco et al., 2017). In particular, the area in the intraparietal sulcus commonly is activated in fMRI activation studies of visual and orthographic tasks (Price and Friston, 1997; Cohen et al., 2004; Purcell et al., 2011; Fairhall and Caramazza, 2013). In a previous activation study of reading in SvPPA, this region showed activation when patients read pseudo-words and made regularization errors in reading real words, thus using sublexical orthography-to-phonology transformations (Wilson et al., 2009). Finally, connectivity metrics in this ICN correlated with pseudoword reading scores in svPPA (Battistella et al., 2019). Further studies are necessary to confirm the role of this ICN in reading and visual attention.

The four networks showed consistent spatial distributions of the functional connectivity maps among two independent groups of controls, thus highlighting the high reproducibility of language 
networks from tf-fMRI data. It is worth noting that the distribution of the task-free networks within the parietal area is relatively segregated anatomically and functionally: Spt and supramarginal areas are part of circuits involved in auditory, phonological, and articulatory aspects of language, whereas the angular gyrus in semantic and comprehension processes. This technique thus can help to better understand the parcellation of the complex inferior parietal region.

Collectively, these findings provide critical support that tffMRI ICNs can serve as a useful tool to study functional connectivity of networks associated with four language functions. These four networks can be derived using a single tf-MRI acquisition without requiring participants to perform four different tasks (1 for each language function), and consequently, this approach would be invaluable within clinical populations for which the execution of language tasks is challenging. Furthermore, we propose that the application of tf-fMRI to studying language disorders will help to elucidate the specific functions of ICNs in neurological patients.

Behavioral neurology has demonstrated that studying languagerelated syndromes can advance the characterization of not only the disorders themselves, but also the neurobiology of language (Hillis et al., 2004; Schwartz et al., 2009, 2012; Dronkers et al., 2017). Previous studies have shown that the anatomical distribution of ICNs identified in HCs map to the pattern of atrophy in specific neurodegenerative syndromes, suggesting that there is a selective vulnerability of specific large-scale networks to neurodegeneration (Seeley et al., 2009; Zhou et al., 2010; Mandelli et al., 2016; Ossenkoppele et al., 2019). These approaches differ from ours, as prior studies chose seeds based on the peak of the atrophy regions in each clinical syndrome, rather than their functional relevance in language function. Furthermore, no previous study has considered the similarity between language ICNs and patterns of atrophy in all three PPA variants. This measure of similarity showed that the speech production and semantic networks were the most representative in nfvPPA and svPPA, respectively. This matches the clear, segregated clinical and linguistic profiles of these two variants. In lvPPA, we did not find a single-most representative ICN, consistent with greater clinical and anatomical heterogeneity in this more recently defined syndrome (Louwersheimer et al., 2016). Instead, all three posterior networks showed a similar degree of overlap with atrophy in the lvPPA group. These findings suggest a possible clinical-anatomical subdivision within the lvPPA syndrome (Ramanan et al., 2019). Overall, our approach of building networks from functionallydefined cortical seeds has the potential to improve the characterization of the interplay between functional anatomy of language and selective vulnerability to neurodegenerative disease.

The study has several limitations. Additional ROIs could have been included, because many left-hemisphere regions are crucial for language. In this initial study, we aimed to identify ICNs that most likely would show segregated patterns of connectivity and consequently behavioral specificity as well. Limitations of the Neurosynth analysis include the choice of key words used to query the database, which impacted the type and number of studies analyzed. To overcome this issue, we chose broad key words, such as speech production, speech perception, semantics, and orthography. Also, although easy to use, Neurosynth does not allow the user to filter the papers entering the meta-analysis to better control for differences in the experimental design (i.e., baseline conditions), thus influencing the activation patterns of the resulting maps. Future studies investigating activation and task-free fMRI in the same subjects will provide more precise characterization of the cognitive functions associated with each tf-fMRI ICNs.

The present study identified four reproducible and segregated task-free networks corresponding to fundamental language functions (i.e., speech production, perception, semantics, and orthography). Our results offer the potential use of a short, tf-fMRI acquisition to investigate functionality of language networks in controls and patients for whom activation studies can be methodologically challenging.

\section{References}

Amici S, Brambati SM, Wilkins DP, Ogar J, Dronkers NL, Miller BL, GornoTempini ML (2007) Anatomical correlates of sentence comprehension and verbal working memory in neurodegenerative disease. J Neurosci 27:6282-6290.

Ashburner J (2007) A fast diffeomorphic image registration algorithm. Neuroimage 38:95-113.

Battistella G, Henry M, Gesierich B, Wilson SM, Borghesani V, Shwe W, Miller Z, Deleon J, Miller BL, Jovicich J, Papinutto N, Dronkers NF, Seeley WW, Mandelli ML, Gorno-Tempini ML (2019) Differential intrinsic functional connectivity changes in semantic variant primary progressive aphasia. Neuroimage Clin 22:101797.

Binder JR, Desai RH (2011) The neurobiology of semantic memory. Trends Cogn Sci 15:527-536.

Binder JR, Frost JA, Hammeke TA, Cox RW, Rao SM, Prieto T (1997) Human brain language areas identified by functional magnetic resonance imaging. J Neurosci 17:353-362.

Binder JR, McKiernan KA, Parsons ME, Westbury CF, Possing ET, Kaufman JN, Buchanan L (2003) Neural correlates of lexical access during visual word recognition. J Cogn Neurosci 15:372-393.

Binder JR, Desai RH, Graves WW, Conant LL (2009) Where is the semantic system? A critical review and meta-analysis of 120 functional neuroimaging studies. Cereb Cortex 19:2767-2796.

Bolger DJ, Minas J, Burman DD, Booth JR (2008) Differential effects of orthographic and phonological consistency in cortex for children with and without reading impairment. Neuropsychologia 46:3210-3224.

Bonilha L, Moser D, Rorden C, Baylis GC, Fridriksson J (2006) Speech apraxia without oral apraxia: can normal brain function explain the physiopathology? Neuroreport 17:1027-1031.

Booth JR, Burman DD, Meyer JR, Gitelman DR, Parrish TB, Mesulam MM (2003) Relation between brain activation and lexical performance. Hum Brain Mapp 19:155-169.

Broca P (1861) Perte de la parole: ramollissement chronique et destruction partielle du lobe anterieur gauche du cerveau. Bulletins de la Societe d'anthropologie 2:235-238.

Büchel C, Price C, Friston K (1998) A multimodal language region in the ventral visual pathway. Nature 394:274-277.

Buchsbaum BR, Baldo J, Okada K, Berman KF, Dronkers N, D’Esposito M, Hickok G (2011) Conduction aphasia, sensory-motor integration, and phonological short-term memory: an aggregate analysis of lesion and fMRI data. Brain Lang 119:119-128.

Butler RA, Lambon Ralph MA, Woollams AM (2014) Capturing multidimensionality in stroke aphasia: mapping principal behavioural components to neural structures. Brain 137:3248-3266.

Catani M, Mesulam MM, Jakobsen E, Malik F, Martersteck A, Wieneke C, Thompson CK, Thiebaut de Schotten M, Dell'Acqua F, Weintraub S, Rogalski E (2013) A novel frontal pathway underlies verbal fluency in primary progressive aphasia. Brain 136:2619-2628.

Cheung C, Hamiton LS, Johnson K, Chang EF (2016) The auditory representation of speech sounds in human motor cortex. eLife 5:e12577.

Cohen L, Jobert A, Le Bihan D, Dehaene S (2004) Distinct unimodal and multimodal regions for word processing in the left temporal cortex. Neuroimage 23:1256-1270.

Corbetta M, Kincade JM, Ollinger JM, McAvoy MP, Shulman GL (2000) Voluntary orienting is dissociated from target detection in human posterior parietal cortex. Nat Neurosci 3:292-297.

Damoiseaux JS, Rombouts SA, Barkhof F, Scheltens P, Stam CJ, Smith SM, Beckmann CF (2006) Consistent resting-state networks across healthy subjects. Proc Natl Acad Sci U S A 103:13848-13853.

DeMarco AT, Wilson SM, Rising K, Rapcsak SZ, Beeson PM (2017) Neural substrates of sublexical processing for spelling. Brain Lang 164:118-128. 
den Ouden DB, Malyutina S, Basilakos A, Bonilha L, Gleichgerrcht E, Yourganov G, Hillis AE, Hickok G, Rorden C, Fridriksson J (2019) Cortical and structural-connectivity damage correlated with impaired syntactic processing in aphasia. Hum Brain Mapp 40:2153-2173.

Dronkers N, Ogar J (2004) Brain areas involved in speech production. Brain 127:1461-1462.

Dronkers NF, Ivanova MV, Baldo JV (2017) What do language disorders reveal about brain-language relationships? from classic models to network approaches. J Int Neuropsychol Soc 23:741-754.

Fairhall SL, Caramazza A (2013) Brain regions that represent amodal conceptual knowledge. J Neurosci 33:10552-10558.

Fedorenko E, Thompson-Schill SL (2014) Reworking the language network. Trends Cogn Sci 18:120-126.

Fox MD, Raichle ME (2007) Spontaneous fluctuations in brain activity observed with functional magnetic resonance imaging. Nat Rev Neurosci 8:700-711.

Fox MD, Corbetta M, Snyder AZ, Vincent JL, Raichle ME (2006) Spontaneous neuronal activity distinguishes human dorsal and ventral attention systems. Proc Natl Acad Sci U S A 103:10046-10051.

Friederici AD, Gierhan SM (2013) The language network. Curr Opin Neurobiol 23:250-254.

Friederici AD, Bahlmann J, Heim S, Schubotz RI, Anwander A (2006) The brain differentiates human and non-human grammars: functional localization and structural connectivity. Proc Natl Acad Sci U S A 103:2458-2463.

Galaburda A, Sanides F (1980) Cytoarchitectonic organization of the human auditory-cortex. J Comp Neurol 190:597-610.

Gesierich B, Jovicich J, Riello M, Adriani M, Monti A, Brentari V, Robinson SD, Wilson SM, Fairhall SL, Gorno-Tempini ML (2012) Distinct neural substrates for semantic knowledge and naming in the temporoparietal network. Cereb Cortex 22:2217-2226.

Gorno-Tempini ML, Miller BL (2013) Primary progressive aphasia as a model to study the neurobiology of language. Brain Lang 127:105.

Gorno-Tempini ML, Price CJ, Josephs O, Vandenberghe R, Cappa SF, Kapur N, Frackowiak RS (1998) The neural systems sustaining face and proper-name processing. Brain 121:2103-2118.

Gorno-Tempini ML, Dronkers NF, Rankin KP, Ogar JM, Phengrasamy L, Rosen HJ, Johnson JK, Weiner MW, Miller BL (2004) Cognition and anatomy in three variants of primary progressive aphasia. Ann Neurol 55:335-346.

Gorno-Tempini ML, Hillis AE, Weintraub S, Kertesz A, Mendez M, Cappa SF, Ogar JM, Rohrer JD, Black S, Boeve BF, Manes F, Dronkers NF, Vandenberghe R, Rascovsky K, Patterson K, Miller BL, Knopman DS, Hodges JR, Mesulam MM, Grossman M (2011) Classification of primary progressive aphasia and its variants. Neurology 76:1006-1014.

Greicius MD, Krasnow B, Reiss AL, Menon V (2003) Functional connectivity in the resting brain: a network analysis of the default mode hypothesis. Proc Natl Acad Sci U S A 100:253-258.

Grossman M (2012) The non-fluent/agrammatic variant of primary progressive aphasia. Lancet Neurol 11:545-555.

Grossman M, Mickanin J, Onishi K, Hughes E, D’Esposito M, Ding XS, Alavi A, Reivich M (1996) Progressive nonfluent aphasia: language, cognitive, and PET measures contrasted with probable Alzheimer's disease. J Cogn Neurosci 8:135-154.

Hallquist MN, Hwang K, Luna B (2013) The nuisance of nuisance regression: spectral misspecification in a common approach to resting-state fMRI preprocessing reintroduces noise and obscures functional connectivity. Neuroimage 82:208-225.

Heim S, Eickhoff SB, Amunts K (2008) Specialisation in Broca's region for semantic, phonological, and syntactic fluency? Neuroimage 40:1362-1368.

Henry ML, Gorno-Tempini ML (2010) The logopenic variant of primary progressive aphasia. Curr Opin Neurol 23:633-637.

Hickok G, Poeppel D (2004) Dorsal and ventral streams: a framework for understanding aspects of the functional anatomy of language. Cognition 92:67-99.

Hickok G, Poeppel D (2007) The cortical organization of speech processing. Nat Rev Neurosci 8:393-402.

Hillis AE, Work M, Barker PB, Jacobs MA, Breese EL, Maurer K (2004) Re-examining the brain regions crucial for orchestrating speech articulation. Brain 127:1479-1487.

Hodges JR, Patterson K, Oxbury S, Funnell E (1992) Semantic dementia: progressive fluent aphasia with temporal lobe atrophy. Brain 115:1783-1806.
Houde JN, Nagarajan S (2015) Auditory feedback. In: The handbook of speech production (Redford MA, ed). Hoboken, NJ: Wiley.

Humphries C, Love T, Swinney D, Hickok G (2005) Response of anterior temporal cortex to syntactic and prosodic manipulations during sentence processing. Hum Brain Mapp 26:128-138.

Hurley RS, Bonakdarpour B, Wang X, Mesulam MM (2015) Asymmetric connectivity between the anterior temporal lobe and the language network. J Cogn Neurosci 27:464-473.

Kuperberg GR, Lakshmanan BM, Greve DN, West WC (2008) Task and semantic relationship influence both the polarity and localization of hemodynamic modulation during lexico-semantic processing. Hum Brain Mapp 29:544-561.

La Joie R, Perrotin A, Barré L, Hommet C, Mézenge F, Ibazizene M, Camus V, Abbas A, Landeau B, Guilloteau D, de La Sayette V, Eustache F, Desgranges B, Chételat G (2012) Region- specific hierarchy between atrophy, hypometabolism, and $\beta$-amyloid $(\mathrm{A} \beta$ ) load in Alzheimer's disease dementia. J Neurosci 32:16265-16273.

Louwersheimer E, Keulen MA, Steenwijk MD, Wattjes MP, Jiskoot LC, Vrenken H, Teunissen CE, van Berckel BN, van der Flier WM, Scheltens P, van Swieten JC, Pijnenburg YA (2016) Heterogeneous language profiles in patients with primary progressive aphasia due to Alzheimer's disease. J Alzheimers Dis 51:581-590.

MacSweeney M, Brammer MJ, Waters D, Goswami U (2009) Enhanced activation of the left inferior frontal gyrus in deaf and dyslexic adults during rhyming. Brain 132:1928-1940.

Maldjian JA, Laurienti PJ, Kraft RA, Burdette JH (2003) An automated method for neuroanatomic and cytoarchitectonic atlas-based interrogation of fMRI data sets. Neuroimage 19:1233-1239.

Mandelli ML, Caverzasi E, Binney RJ, Henry ML, Lobach I, Block N, Amirbekian B, Dronkers N, Miller BL, Henry RG, Gorno-Tempini ML (2014) Frontal white matter tracts sustaining speech production in primary progressive aphasia. J Neurosci 34:9754-9767.

Mandelli ML, Vilaplana E, Brown JA, Hubbard HI, Binney RJ, Attygalle S, Santos-Santos MA, Miller ZA, Pakvasa M, Henry ML, Rosen HJ, Henry RG, Rabinovici GD, Miller BL, Seeley WW, Gorno-Tempini ML (2016) Healthy brain connectivity predicts atrophy progression in non-fluent variant of primary progressive aphasia. Brain 139:2778-2791.

Matsuo K, Chen SH, Tseng WY (2012) AveLI: a robust lateralization index in functional magnetic resonance imaging using unbiased threshold-free computation. J Neurosci Methods 205:119-129.

Mechelli A, Josephs O, Lambon Ralph MA, McClelland JL, Price CJ (2007) Dissociating stimulus-driven semantic and phonological effect during reading and naming. Hum Brain Mapp 28:205-217.

Mesulam MM (1990) Large-scale neurocognitive networks and distributed processing for attention, language, and memory. Ann Neurol 28:597-613.

Milner AD, Goodale MA (1995) The visual brain in action. Oxford; New York: Oxford UP.

Montembeault M, Chapleau M, Jarret J, Boukadi M, Laforce R Jr, Wilson MA, Rouleau I, Brambati SM (2019) Differential language network functional connectivity alterations in Alzheimer's disease and the semantic variant of primary progressive aphasia. Cortex 117:284-298.

Möttönen R, Dutton R, Watkins KE (2013) Auditory-motor processing of speech sounds. Cereb Cortex 23:1190-1197.

Mummery CJ, Patterson K, Wise RJ, Vandenberghe R, Price CJ, Hodges JR (1999) Disrupted temporal lobe connections in semantic dementia. Brain 122:61-73.

Okada K, Hickok G (2006) Left posterior auditory-related cortices participate both in speech perception and speech production: neural overlap revealed by fMRI. Brain Lang 98:112-117.

Ossenkoppele R, Cohn-Sheehy BI, La Joie R, Vogel JW, Möller C, Lehmann M, van Berckel BN, Seeley WW, Pijnenburg YA, Gorno-Tempini ML, Kramer JH, Barkhof F, Rosen HJ, van der Flier WM, Jagust WJ, Miller BL, Scheltens P, Rabinovici GD (2015) Atrophy patterns in early clinical stages across distinct phenotypes of Alzheimer's disease. Hum Brain Mapp 36:4421-4437.

Ossenkoppele R, Iaccarino L, Schonhaut DR, Brown JA, La Joie R, O’Neil JP, Janabi M, Baker SL, Kramer JH, Gorno-Tempini ML, Miller BL, Rosen HJ, Seeley WW, Jagust WJ, Rabinovici GD (2019) Tau covariance patterns in Alzheimer's disease patients match intrinsic connectivity networks in the healthy brain. Neuroimage Clin 23:101848.

Pa J, Hickok G (2008) A parietal-temporal sensory-motor integration area 
for the human vocal tract: evidence from an fMRI study of skilled musicians. Neuropsychologia 46:362-368.

Price CJ (2000) The anatomy of language: contributions from functional neuroimaging. J Anat 197:335-359.

Price CJ (2012) A review and synthesis of the first 20 years of PET and fMRI studies of heard speech, spoken language and reading. Neuroimage 62:816-847.

Price CJ, Friston KJ (1997) The temporal dynamics of reading: a PET study. Proc Biol Sci 264:1785-1791.

Price CJ, Crinion J, Friston KJ (2006a) Design and analysis of fMRI studies with neurologically impaired patients. J Magn Reson Imaging 23:816-826.

Price CJ, McCrory E, Noppeney U, Mechelli A, Moore CJ, Biggio N, Devlin JT (2006b) How reading differs from object naming at the neuronal level. Neuroimage 29:643-648.

Purcell JJ, Turkeltaub PE, Eden GF, Rapp B (2011) Examining the central and peripheral processes of written word production through metaanalysis. Front Psychol 2:239.

Raichle ME (2006) Neuroscience. the brain's dark energy. Science 314:1249-1250.

Ralph MA, Jefferies E, Patterson K, Rogers TT (2017) The neural and computational bases of semantic cognition. Nat Rev Neurosci 18:42-55.

Ramanan SR, Roquet D, Goldberg Z, Hodges JR, Piguet O, Irish M, Lambon Ralph MA (2019) Establishing two principal dimensions of cognitive variation in logopenic progressive aphasia. bioRxiv 767269 .

Satterthwaite TD, Elliott MA, Gerraty RT, Ruparel K, Loughead J, Calkins ME, Eickhoff SB, Hakonarson H, Gur RC, Gur RE, Wolf DH (2013) An improved framework for confound regression and filtering for control of motion artifact in the preprocessing of resting-state functional connectivity data. Neuroimage 64:240-256.

Schwartz MF, Kimberg DY, Walker GM, Faseyitan O, Brecher A, Dell GS, Coslett HB (2009) Anterior temporal involvement in semantic word retrieval: voxel-based lesion-symptom mapping evidence from aphasia. Brain 132:3411-3427.

Schwartz MF, Faseyitan O, Kim J, Coslett HB (2012) The dorsal stream contribution to phonological retrieval in object naming. Brain 135:3799-3814.

Seeley WW, Crawford RK, Zhou J, Miller BL, Greicius MD (2009) Neurodegenerative diseases target large-scale human brain networks. Neuron 62:42-52.

Seghier ML, Fagan E, Price CJ (2010) Functional subdivisions in the left angular gyrus where the semantic system meets and diverges from the default network. J Neurosci 30:16809-16817.

Shehzad Z, Kelly AM, Reiss PT, Gee DG, Gotimer K, Uddin LQ, Lee SH, Margulies DS, Roy AK, Biswal BB, Petkova E, Castellanos FX, Milham MP (2009) The resting brain: unconstrained yet reliable. Cereb Cortex 19:2209-2229.

Simmons WK, Reddish M, Bellgowan PS, Martin A (2010) The selectivity and functional connectivity of the anterior temporal lobes. Cereb Cortex 20:813-825.

Smith SM, Fox PT, Miller KL, Glahn DC, Fox PM, Mackay CE, Filippini N, Watkins KE, Toro R, Laird AR, Beckmann CF (2009) Correspondence of the brain's functional architecture during activation and rest. Proc Natl Acad Sci U S A 106:13040-13045.

Tomasi D, Volkow ND (2012) Resting functional connectivity of language networks: characterization and reproducibility. Mol Psychiatry 17:841-854.

Turken AU, Dronkers NF (2011) The neural architecture of the language comprehension network: converging evidence from lesion and connectivity analyses. Front Syst Neurosci 5:1.

Van Dijk KR, Hedden T, Venkataraman A, Evans KC, Lazar SW, Buckner RL (2010) Intrinsic functional connectivity as a tool for human connectomics: theory, properties, and optimization. J Neurophysiol 103:297-321.

Wilson SM, Saygin AP, Sereno MI, Iacoboni M (2004) Listening to speech activates motor areas involved in speech production. Nat Neurosci 7:701-702.

Wilson SM, Brambati SM, Henry RG, Handwerker DA, Agosta F, Miller BL, Wilkins DP, Ogar JM, Gorno-Tempini ML (2009) The neural basis of surface dyslexia in semantic dementia. Brain 132:71-86.

Wilson SM, Galantucci S, Tartaglia MC, Rising K, Patterson DK, Henry ML, Ogar JM, DeLeon J, Miller BL, Gorno-Tempini ML (2011) Syntactic processing depends on dorsal language tracts. Neuron 72:397-403.

Yarkoni T, Poldrack RA, Nichols TE, Van Essen DC, Wager TD (2011) Large-scale automated synthesis of human functional neuroimaging data. Nat Methods 8:665-670.

Zhou J, Greicius MD, Gennatas ED, Growdon ME, Jang JY, Rabinovici GD, Kramer JH, Weiner M, Miller BL, Seeley WW (2010) Divergent network connectivity changes in behavioural variant frontotemporal dementia and Alzheimer's disease. Brain 133:1352-1367. 